\title{
Adaptive Minimum Bit Error Rate Beamforming Assisted QPSK Receiver
}

\author{
S. Chen, L. Hanzo, N.N. Ahmad and A. Wolfgang \\ School of Electronics and Computer Science \\ University of Southampton \\ Southampton SO17 1BJ, U.K. \\ E-mails: $\{$ sqc,lh, nna00r,aw03r\}@ecs.soton.ac.uk \\ Presented at IEEE International Conference on Communications \\ Paris, France, June 20-24, 2004
}




\section{Overview}

Adaptive beamforming assisted multiuser detection for multiple receive antennas aided SDMA systems with QPSK modulation scheme

Motivation for minimum bit error rate design

$\bigcirc$ System model and standard minimum mean square error solution

Minimum bit error rate beamforming solution

Adaptive implementation of minimum bit error rate design

Simulation results 


\section{Motivation}

128-subcarriers OFDM 4-receive-antennas aided SDMA, observing user 1 BER with increasing number of users:

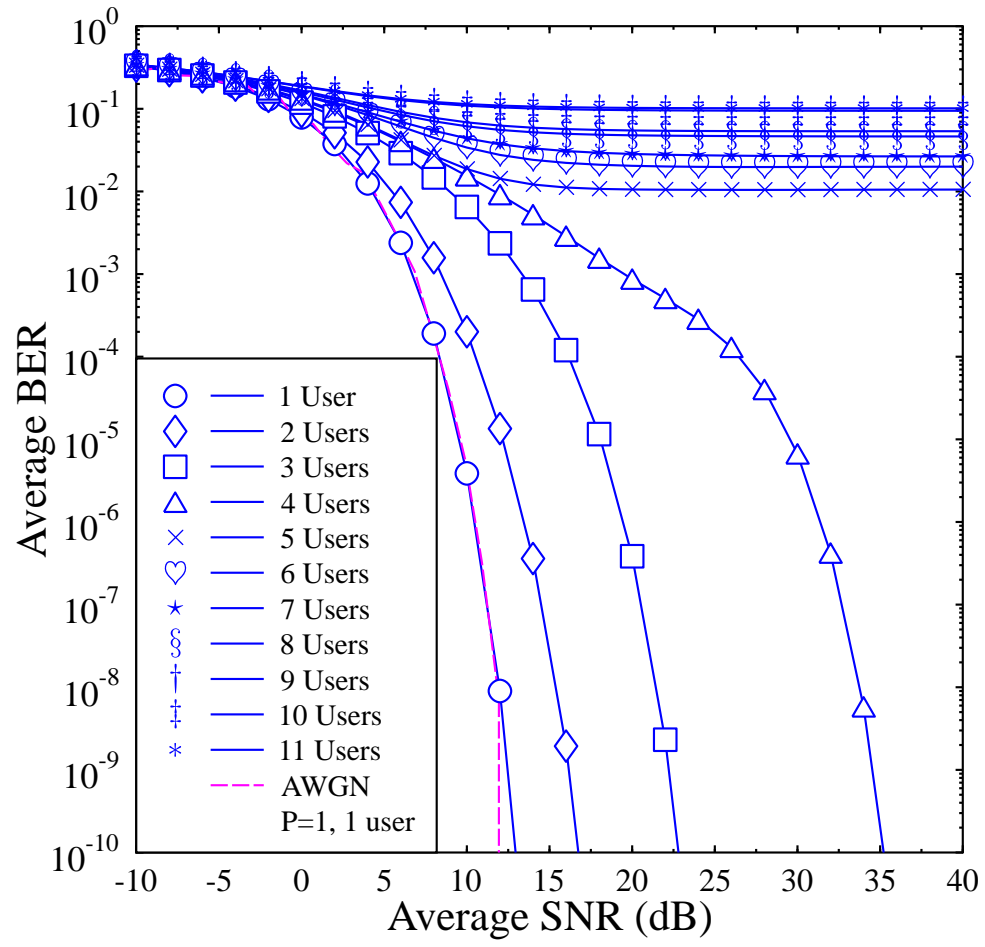

(a) MMSE

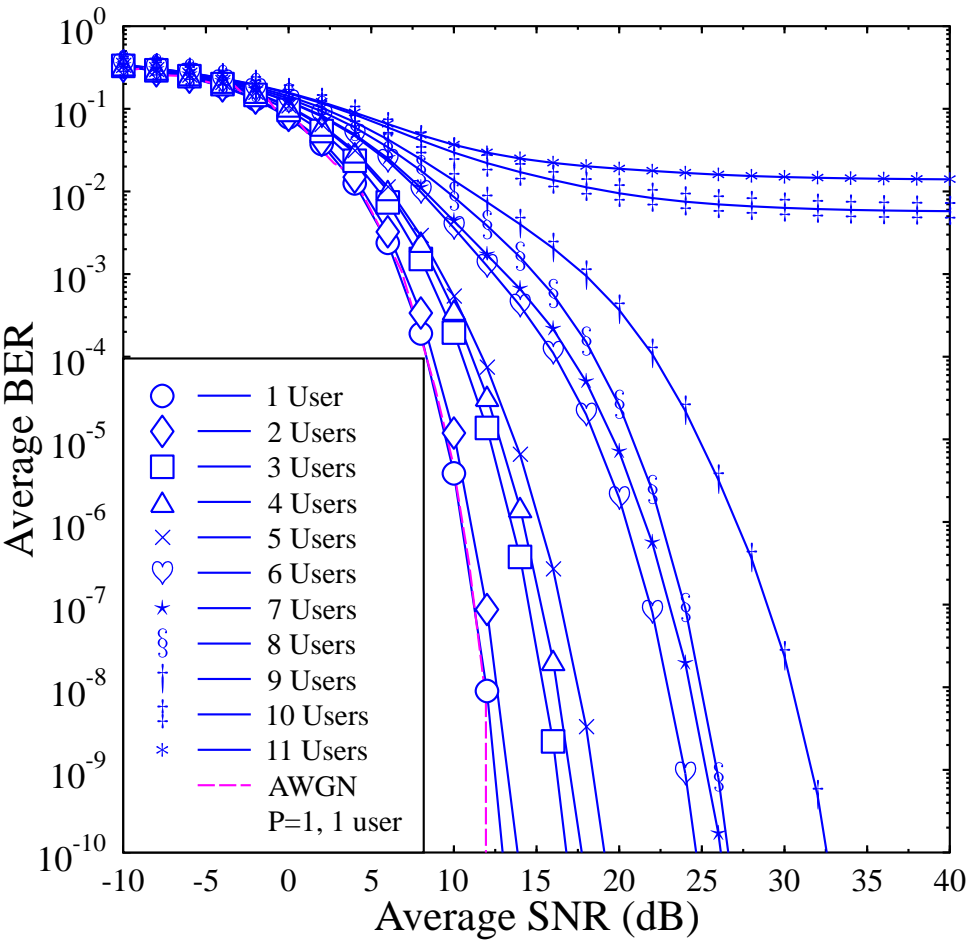

(b) MBER

Given number of antennas, capacity is fixed. But changing design from MMSE to MBER $\Rightarrow$ improve performance or better realizing the capacity 


\section{System Model}

$\bigcirc L$ receive antennas and $M$ users, point-source model with narrow band channels $A_{i}$ for $1 \leq i \leq M$

Received signal model:

$$
\mathbf{x}(k)=\overline{\mathbf{x}}(k)+\mathbf{n}(k)=\mathbf{P b}(k)+\mathbf{n}(k)
$$

where $\mathbf{n}(k)=\left[n_{1}(k) \cdots n_{L}(k)\right]^{T}$ is

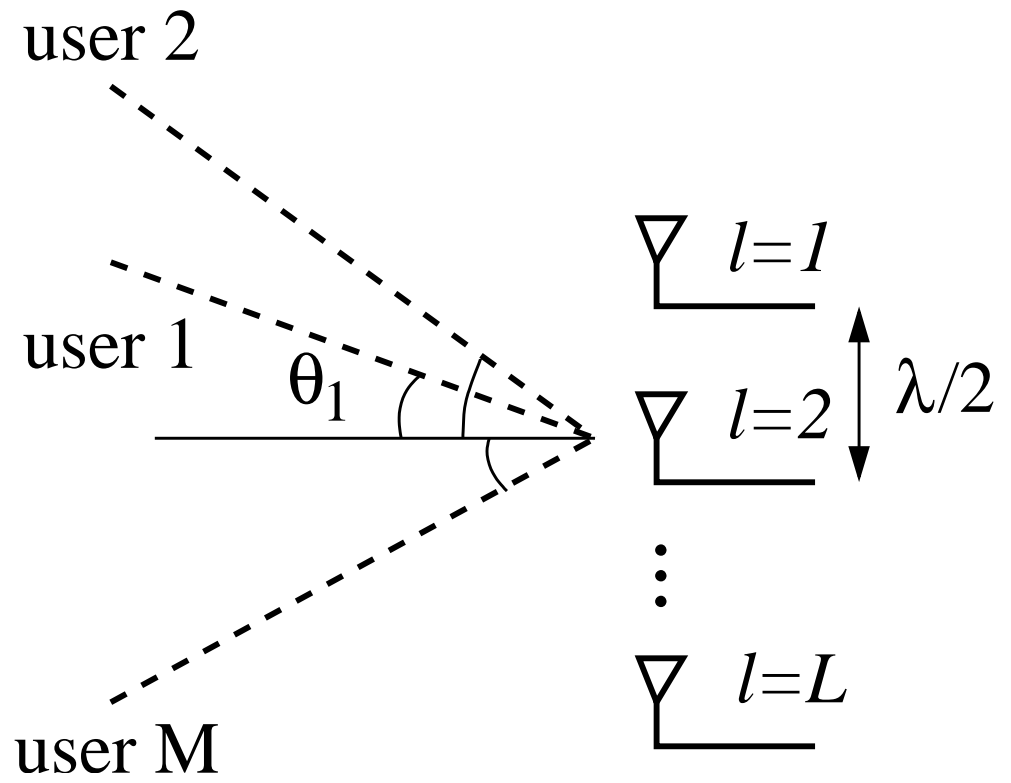
system noise vector, user QPSK symbol vector $\mathbf{b}(k)=\left[b_{1}(k) \cdots b_{M}(k)\right]^{T}$, and system matrix

$$
\mathbf{P}=\left[\begin{array}{lll}
\alpha_{1} A_{1} \mathbf{s}_{1} & \alpha_{2} A_{2} \mathbf{s}_{2} \cdots \alpha_{M} A_{M} \mathbf{s}_{M}
\end{array}\right]
$$

with $\mathbf{s}_{i}, 1 \leq i \leq M$, denoting steering vectors and $\alpha_{i}^{2}$ transmitted signal powers. User 1 is desired user 


\section{Beamforming}

Linear beamformer:

$$
y(k)=\mathbf{w}^{H} \mathbf{x}(k)=\mathbf{w}^{H}(\overline{\mathbf{x}}(k)+\mathbf{n}(k))=\bar{y}(k)+e(k)
$$

with beamformer weight vector $\mathbf{w}=\left[\begin{array}{lll}w_{1} & w_{2} \cdots w_{L}\end{array}\right]^{T}$, and the decision for $b_{1}(k)$ :

$$
\hat{b}_{1}(k)=\operatorname{sgn}\left(y_{R}(k)\right)+j \operatorname{sgn}\left(y_{I}(k)\right)
$$

$\bigcirc$ Let $\mathbf{w}^{H} \mathbf{P}=\mathbf{w}^{H}\left[\mathbf{p}_{1} \mathbf{p}_{2} \cdots \mathbf{p}_{M}\right]=\left[\begin{array}{llll}c_{1} & c_{2} \cdots c_{M}\end{array}\right]$. Then

$$
y(k)=c_{1} b_{1}(k)+\sum_{i=2}^{M} c_{i} b_{i}(k)+e(k)
$$

To make sure $c_{1}$ being real and positive, weight vector rotation:

$$
\mathbf{w}^{\text {new }}=\frac{c_{1}^{\text {old }}}{\left|c_{1}^{\text {old }}\right|} \mathbf{w}^{\text {old }}
$$

$\bigcirc$ Minimum mean square solution: $\mathbf{w}_{\mathrm{MMSE}}=\left(\mathbf{P} \mathbf{P}^{H}+\sigma_{n}^{2} \mathbf{I}_{L}\right)^{-1} \mathbf{p}_{1}, \sigma_{n}^{2}$ being system noise variance and $\mathbf{I}_{L}$ identity matrix 


\section{Bit Error Rate}

Conditional PDF of $y(k)$ given $b_{1}(k)=+1+j$ :

$$
p(y \mid+1+j)=\frac{1}{N_{s b}} \sum_{\bar{y}^{(q)} \in \mathcal{Y}_{+,+}} \frac{1}{2 \pi \sigma_{n}^{2} \mathbf{w}^{H} \mathbf{w}} \exp \left(-\frac{\left|y-\bar{y}^{(q)}\right|^{2}}{2 \sigma_{n}^{2} \mathbf{w}^{H} \mathbf{w}}\right)
$$

where $\bar{y}^{(q)} \in \mathcal{Y}_{+,+}$are points of $\bar{y}(k)$ conditioned on $b_{1}(k)=+1+j$ and $N_{s b}$ is number of points in $\mathcal{Y}_{+,+}$

BER:

$$
P_{E}(\mathbf{w})=\frac{1}{2}\left(P_{E_{R}}(\mathbf{w})+P_{E_{I}}(\mathbf{w})\right)
$$

with

$$
\begin{gathered}
P_{E_{R}}(\mathbf{w})=\frac{1}{N_{s b}} \sum_{\bar{y}^{(q)} \in \mathcal{Y}_{+,+}} Q\left(g_{R}^{(q)}(\mathbf{w})\right) \quad P_{E_{I}}(\mathbf{w})=\frac{1}{N_{s b}} \sum_{\bar{y}^{(q)} \in \mathcal{Y}_{+,+}} Q\left(g_{I}^{(q)}(\mathbf{w})\right) \\
g_{R}^{(q)}(\mathbf{w})=\frac{\operatorname{sgn}\left(b_{R, 1}^{(q)}\right) \bar{y}_{R}^{(q)}}{\sigma_{n} \sqrt{\mathbf{w}^{H} \mathbf{w}}} \quad g_{I}^{(q)}(\mathbf{w})=\frac{\operatorname{sgn}\left(b_{I, 1}^{(q)}\right) \bar{y}_{I}^{(q)}}{\sigma_{n} \sqrt{\mathbf{w}^{H} \mathbf{w}}}
\end{gathered}
$$




\section{Minimum Bit Error Rate}

MBER solution:

$$
\mathbf{w}_{\text {MBER }}=\arg \min _{\mathbf{w}} P_{E}(\mathbf{w})
$$

No closed-from solution, but it can be obtained via gradient-based optimization, with gradient for normalized $\mathbf{w}$ given by

$$
\begin{gathered}
\nabla P_{E}(\mathbf{w})=\frac{1}{2}\left(\nabla P_{E_{R}}(\mathbf{w})+\nabla P_{E_{I}}(\mathbf{w})\right) \\
\nabla P_{E_{R}}(\mathbf{w})=\frac{1}{2 N_{s b} \sqrt{2 \pi} \sigma_{n}} \sum_{\bar{y}(q) \in \mathcal{Y}_{+,+}} \exp \left(-\frac{\left(\bar{y}_{R}^{(q)}\right)^{2}}{2 \sigma_{n}^{2}}\right) \operatorname{sgn}\left(b_{R, 1}^{(q)}\right)\left(\bar{y}_{R}^{(q)} \mathbf{w}-\overline{\mathbf{x}}^{(q)}\right) \\
\nabla P_{E_{I}}(\mathbf{w})=\frac{1}{2 N_{s b} \sqrt{2 \pi} \sigma_{n}} \sum_{\bar{y}^{(q)} \in \mathcal{Y}_{+,+}} \exp \left(-\frac{\left(\bar{y}_{I}^{(q)}\right)^{2}}{2 \sigma_{n}^{2}}\right) \operatorname{sgn}\left(b_{I, 1}^{(q)}\right)\left(\bar{y}_{I}^{(q)} \mathbf{w}+j \overline{\mathbf{x}}^{(q)}\right)
\end{gathered}
$$




\section{Adaptive Implementation}

$\bigcirc$ Given a block of training data $\left\{\mathbf{x}(k), b_{1}(k)\right\}_{k=1}^{K}$, a Parzen window estimate for the PDF of $y(k), p(y)$, is given by

$$
\hat{p}(y)=\frac{1}{K 2 \pi \rho_{n}^{2}} \sum_{k=1}^{K} \exp \left(-\frac{|y-y(k)|^{2}}{2 \rho_{n}^{2}}\right)
$$

where $\rho_{n}$ is kernel width

$\bigcirc$ From the estimated PDF $\hat{p}(y)$, one obtains the estimated BER $\hat{P}_{E}(\mathbf{w})$

Block-data based adaptive MBER solution: minimizing $\hat{P}_{E}(\mathbf{w})$ using a gradientbased optimization

To derive sample-by-sample adaptation, consider one-sample PDF "estimate":

$$
\hat{p}(y, k)=\frac{1}{2 \pi \rho_{n}^{2}} \exp \left(-\frac{|y-y(k)|^{2}}{2 \rho_{n}^{2}}\right)
$$




\section{Least Bit Error Rate}

Conceptually, from one-sample estimate $\hat{p}(y, k)$, one has instantaneous BER $\hat{P}_{E}(\mathbf{w}, k)$

Minimizing this instantaneous BER with stochastic gradient

$$
\nabla \hat{P}_{E}(\mathbf{w}, k)=\frac{\left(-\operatorname{sgn}\left(b_{R, 1}(k)\right) \exp \left(-\frac{y_{R}^{2}(k)}{2 \rho_{n}^{2}}\right)+j \operatorname{sgn}\left(b_{I, 1}(k)\right) \exp \left(-\frac{y_{I}^{2}(k)}{2 \rho_{n}^{2}}\right)\right)}{4 \sqrt{2 \pi} \rho_{n}} \mathbf{x}(k)
$$

leads to the LBER:

$$
\begin{aligned}
\mathbf{w}(k+1) & =\mathbf{w}(k)+\mu\left(-\nabla \hat{P}_{E}(\mathbf{w}(k), k)\right) \\
c_{1} & =\mathbf{w}^{H}(k+1) \mathbf{p}_{1} \\
\mathbf{w}(k+1) & =\frac{c_{1}}{\left|c_{1}\right|} \mathbf{w}(k+1)
\end{aligned}
$$




\section{Comparison with Least Mean Square}

Compared with LMS:

$$
\begin{aligned}
\mathbf{w}(k+1) & =\mathbf{w}(k)+\mu\left(b_{1}(k)-y(k)\right)^{*} \mathbf{x}(k) \\
c_{1} & =\mathbf{w}^{H}(k+1) \mathbf{p}_{1} \\
\mathbf{w}(k+1) & =\frac{c_{1}}{\left|c_{1}\right|} \mathbf{w}(k+1)
\end{aligned}
$$

LBER has a similarly low complexity:

\begin{tabular}{c|c|c|c|c}
\hline algorithm & multiplications & additions & square root & $\exp (\bullet)$ \\
\hline LMS & $16 \times L+6$ & $14 \times L-2$ & 1 & - \\
LBER & $16 \times L+10$ & $14 \times L-4$ & 1 & 2 \\
\hline
\end{tabular}




\section{Simulation (Fixed Channels)}

3-element antenna array, 4 users, and fixed channel conditions: $A_{i}=1+j 0$ for $1 \leq i \leq 4$

Desired user signal to noise ratio:

$$
\mathrm{SNR}=\frac{1}{\sigma_{n}^{2}}
$$

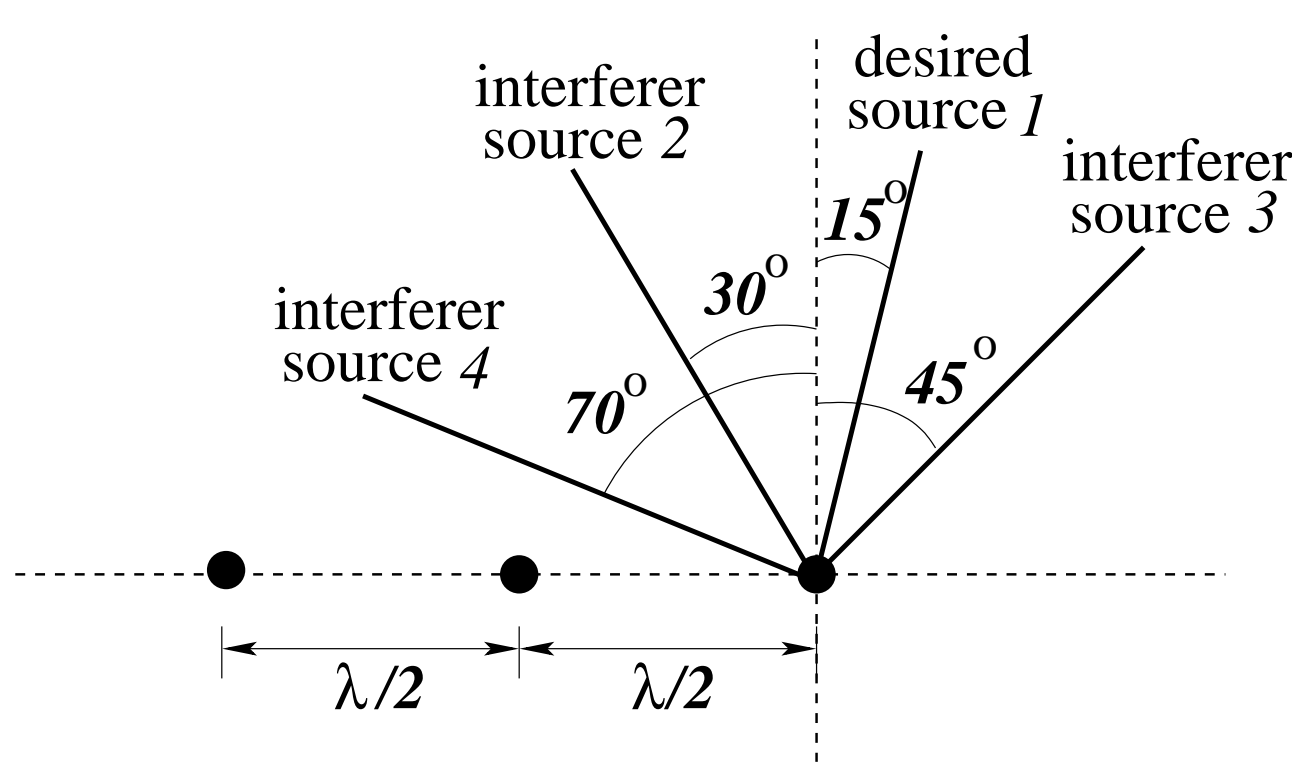

Desired user signal to interferer $i$ ratio:

$$
\mathrm{SIR}_{i}=\frac{\alpha_{1}^{2}}{\alpha_{i}^{2}}, \quad i=2,3,4
$$




\section{Comparison of BERs}

(a) desired user and all three interfering users had equal power: $\mathrm{SIR}_{i}=0 \mathrm{~dB}, i=2,3,4$

(b) desired user and interfering users 2 and 3 had equal power but interfering user 4 had $6 \mathrm{~dB}$ more power than desired user: $\mathrm{SIR}_{2}=0 \mathrm{~dB}, \mathrm{SIR}_{3}=0 \mathrm{~dB}, \mathrm{SIR}_{4}=-6 \mathrm{~dB}$

(c) all three interfering users had $2 \mathrm{~dB}$ more power than desired user: $\mathrm{SIR}_{i}=-2 \mathrm{~dB}, i=2,3,4$

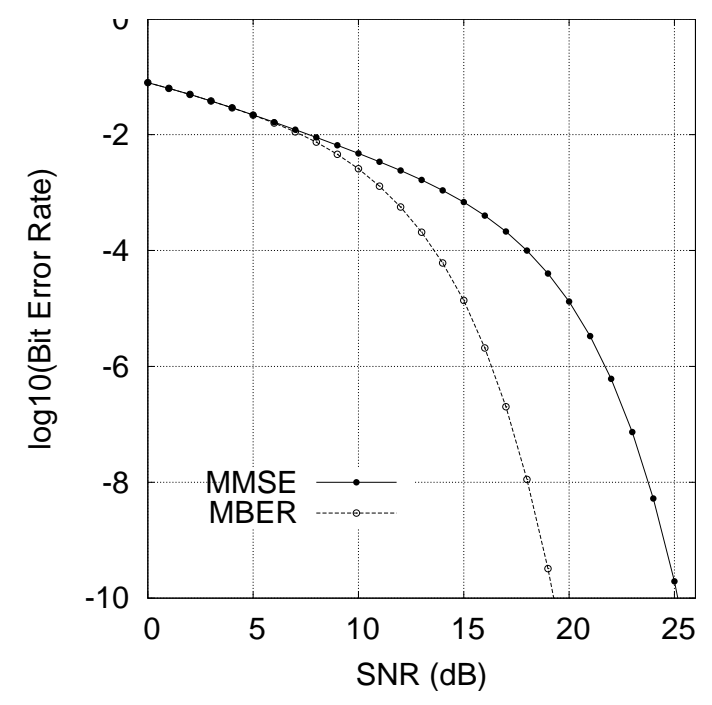

(a)

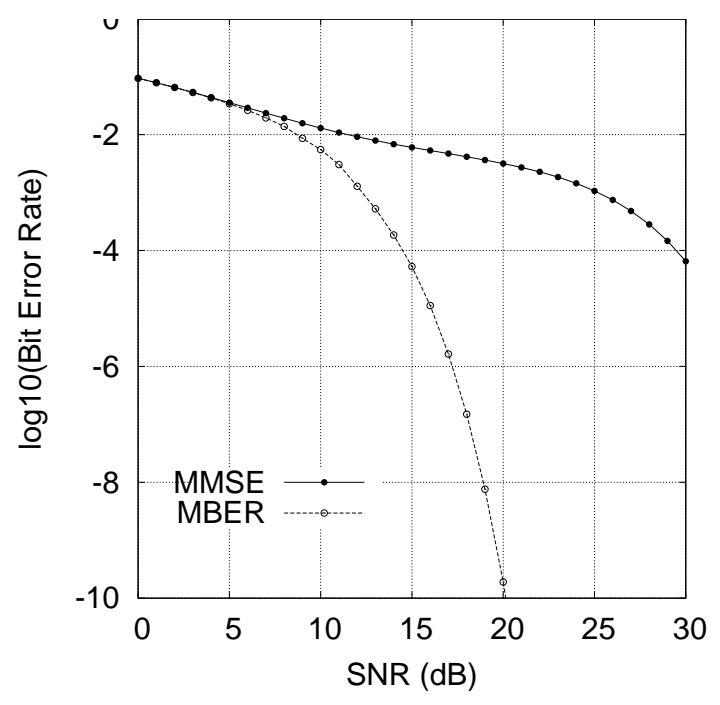

(b)

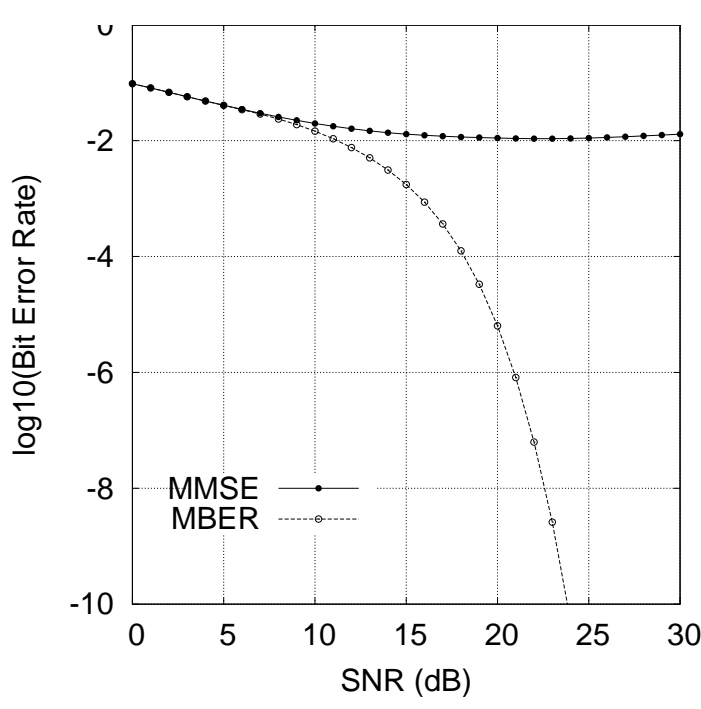

(c) 


\section{Comparison of PDFs}

Case (a) with $\mathrm{SNR}=15 \mathrm{~dB}$ : conditional PDFs, marginal conditional PDFs, and signal subsets $\mathcal{Y}_{+,+}$

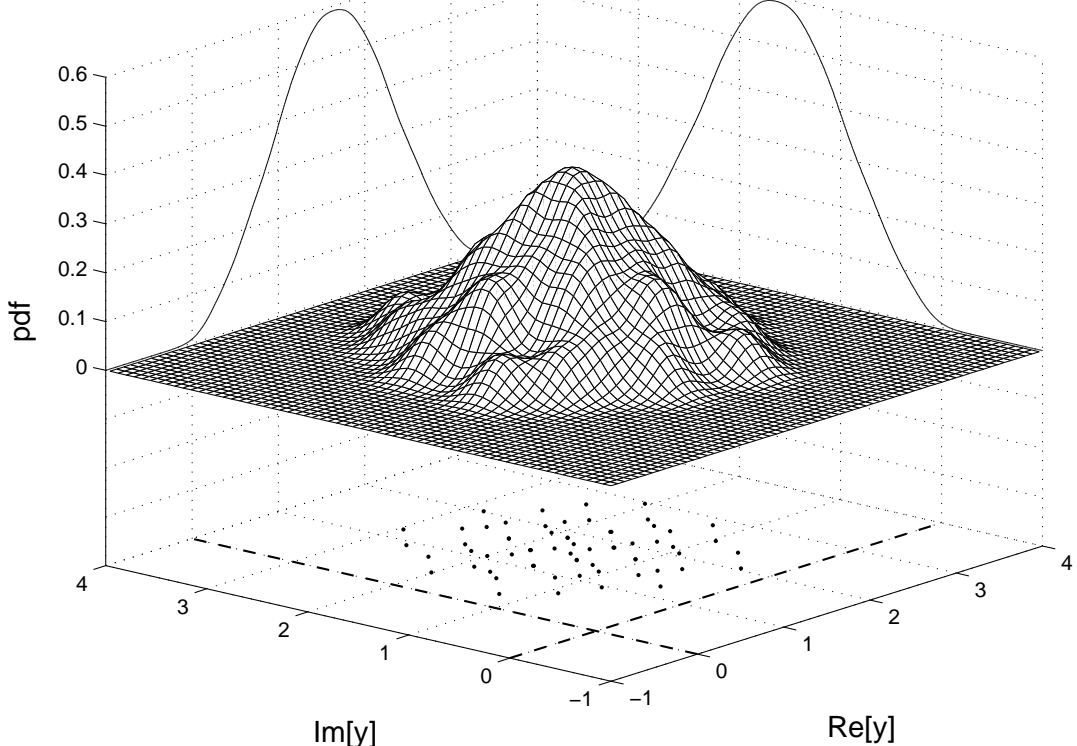

MMSE

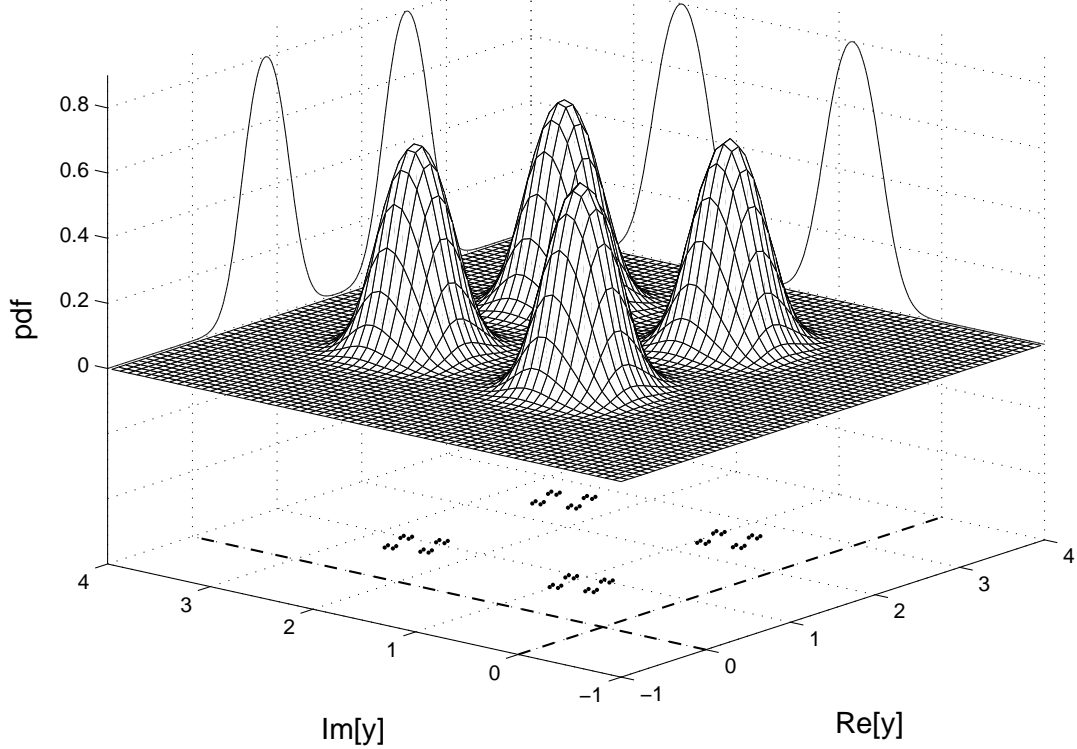

MBER 


\section{Comparison of PDFs}

Case (c) with $\mathrm{SNR}=20 \mathrm{~dB}$ : conditional PDFs, marginal conditional PDFs, and signal subsets $\mathcal{Y}_{+,+}$

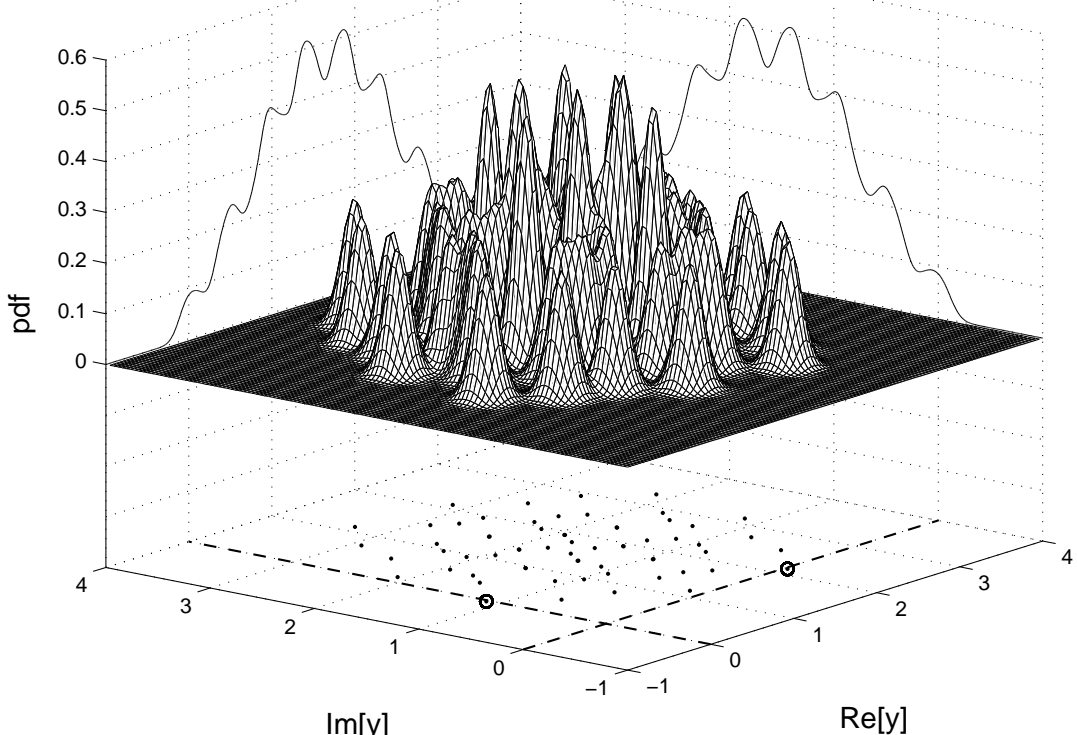

MMSE

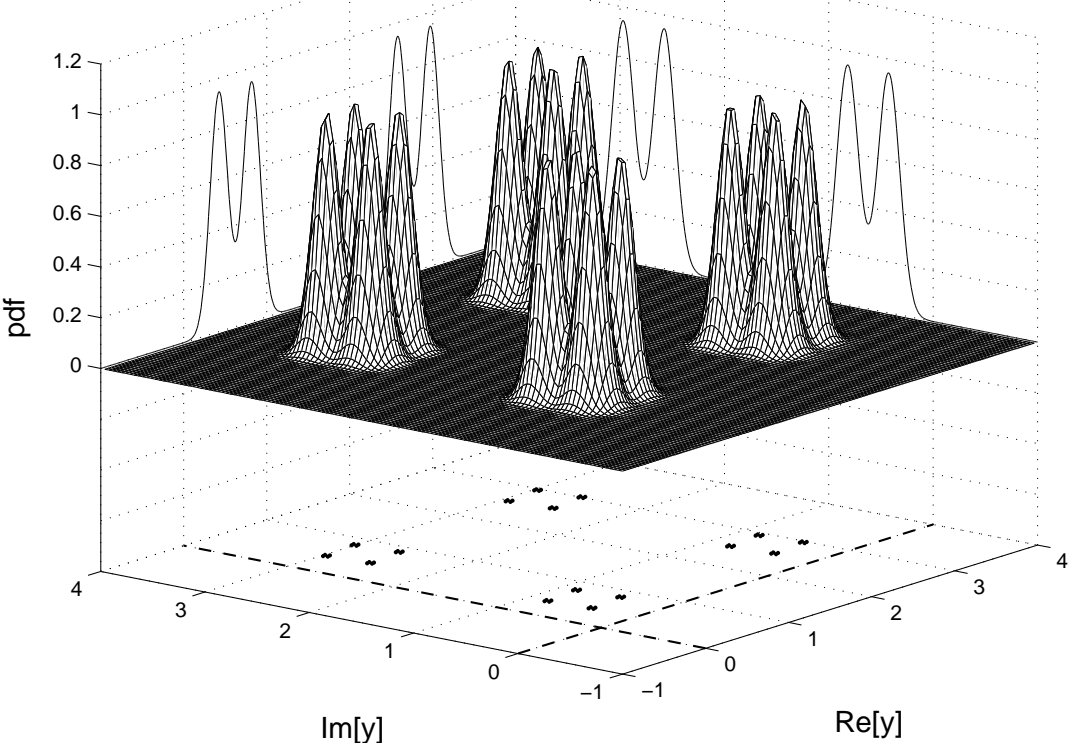

MBER 


\section{Learning Curves of LBER}

Case (b) with SNR $=17 \mathrm{~dB}$ : (i) $\mathbf{w}(0)=\mathbf{w}_{\mathrm{MMSE}}$, and

(ii) $\mathbf{w}(0)=[0.0+j 0.10 .1+j 0.00 .1+j 0.0]^{T}$

DD: decision-directed adaptation with $\hat{b}_{1}(k)$ substituting $b_{1}(k)$

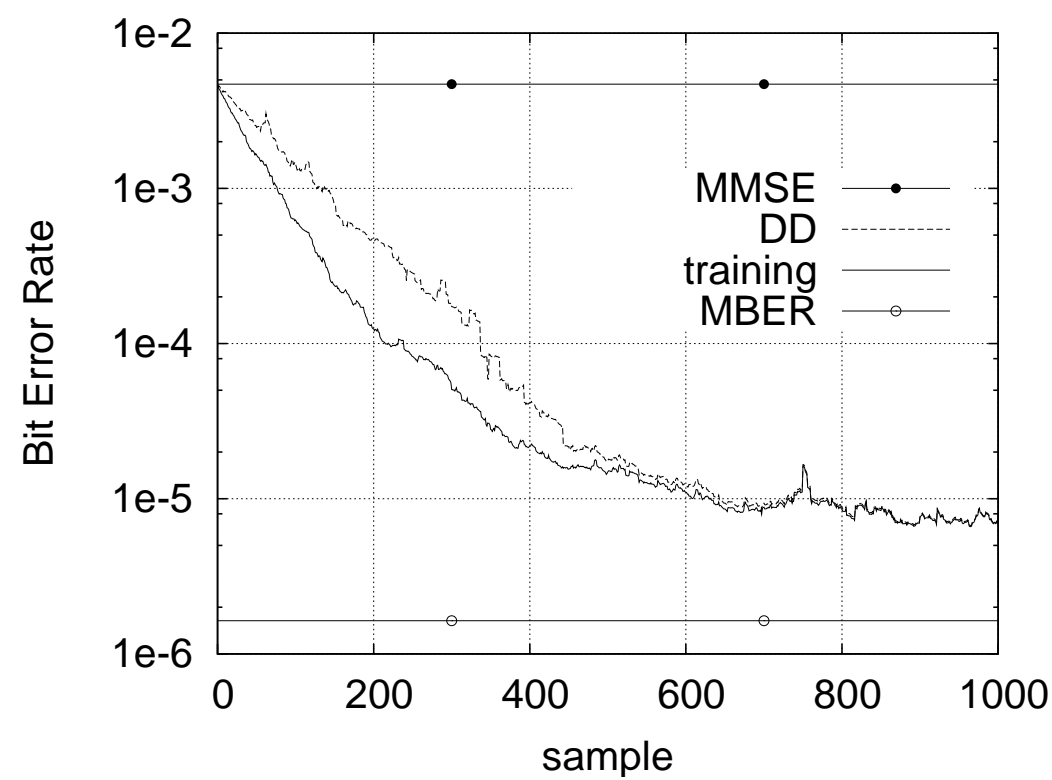

(i)

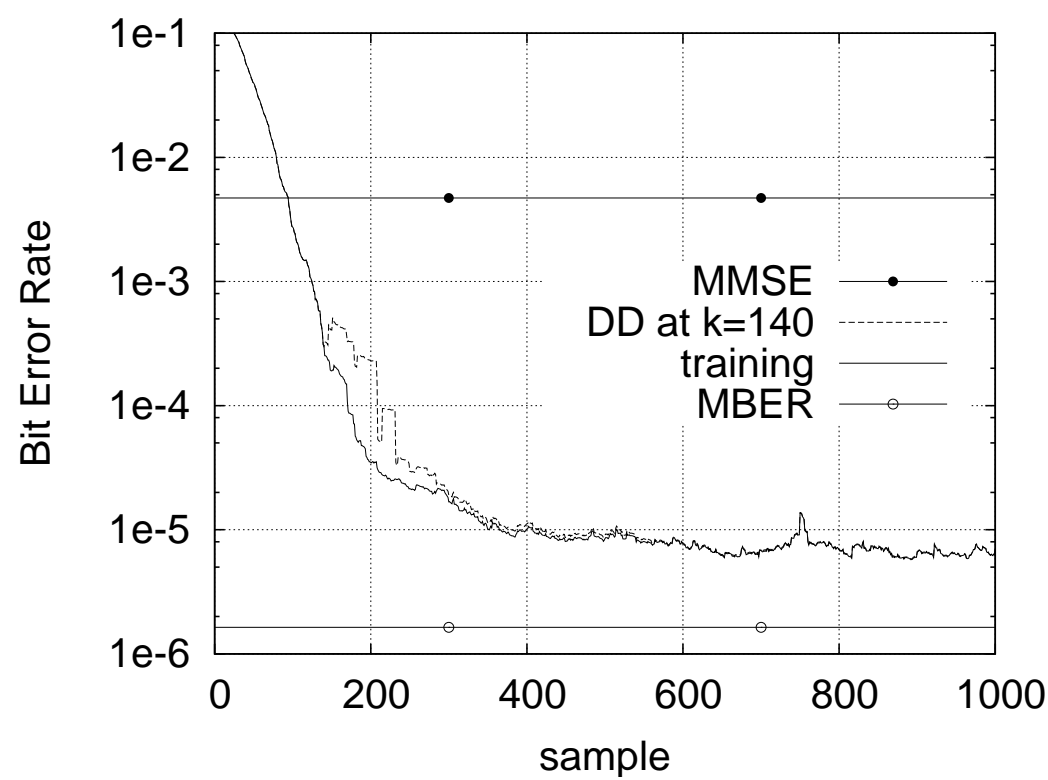

(ii) 


\section{Simulation (Fading Channels)}

Same 3-element antenna array and 4 users, but magnitudes of channels $A_{i}, \quad 1 \leq i \leq 4$, were Rayleigh processes, each with root mean power $\sqrt{0.5}+j \sqrt{0.5}$

Fading was continuous, yielding different channel magnitude and phase for each transmitted symbol

Fading is slow at normalized Doppler frequency $10^{-6}$

Frame structure: 40 training symbols followed by 400 data

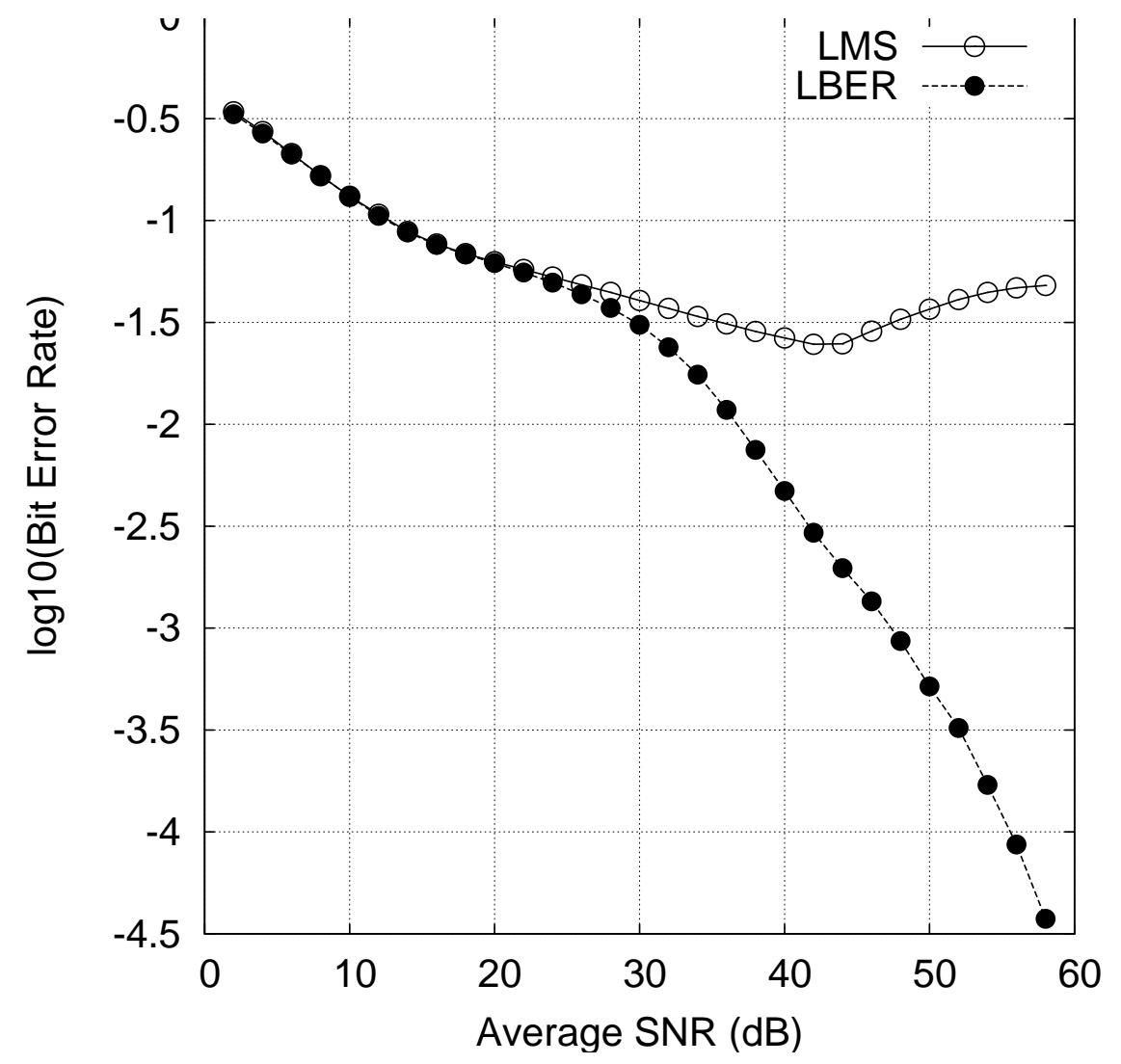
symbols 


\section{Conclusions}

- An adaptive beamforming assisted multiuser detection scheme based on the minimum bit error rate design has been derived for multiple receive antennas aided SDMA systems

- The minimum bit error rate design provides better performance and improves system capacity, compared with the standard minimum mean square error design

- Sample-by-sample adaptation has been realized using the least bit error rate algorithm, which has a similarly low complexity as the least mean square algorithm, for the QPSK modulation

- Our approach can be extended to space-time multiuser detection scheme for generic SDMA systems with wideband channels 